\title{
Removing Elastic Scattering Effects from Chemical Maps Taken under Incoherent Conditions
}

\author{
Y Zhu ${ }^{1}, \mathrm{H} \mathrm{Tan}^{2}, \mathrm{HL} \mathrm{Xin}^{3}$, A Soukiassian ${ }^{4}$, DG Schlom ${ }^{4}$, DA Muller ${ }^{5}$ and $\underline{\mathrm{C} \text { Dwyer }^{6}}$
}

1. Monash Centre for Electron Microscopy, Monash University, Victoria 3800, Australia

2. CEMES-CNRS, Universite' de Toulouse, nMat Group, BP94347, 31055, Toulouse Cedex 4, France

3. Center for Functional Nanomaterials, Brookhaven National Laboratory, Upton, New York 11973, USA

4. Department of Materials Science and Engineering, Cornell University, Ithaca, New York 14853, USA

5. School of Applied and Engineering Physics, Cornell University, Ithaca, New York 14853, USA

6. Ernst Ruska-Centre for Microscopy and Spectroscopy with Electrons, Peter Gruenberg Institute, Forschungszentrum Juelich, Juelich 52425, Germany

Incoherent imaging conditions in scanning transmission electron microscopy (STEM) are generally highly favorable for image interpretation, since the image contrast tends to a slowly-varying (often monotonically) function of specimen thickness and defocus (over reasonable values). Here we define an incoherent condition as that under which the signal (IBF, ADF, EELS, EDX, etc.) is accurately given by multiplying the probe intensity inside the specimen by some object function. For EELS, these conditions are largely satisfied by using a collection angle significantly larger than the probe convergence angle (e.g., $\mathrm{E}_{0}=100 \mathrm{keV}, \alpha \sim 30 \mathrm{mrad}, \beta \sim 80 \mathrm{mrad}$ ).

Nonetheless, even under these highly favorable conditions, strong elastic and thermal diffuse scattering can still cause the EELS signal to be "lost" outside the detector, making image interpretation non-trivial. This occurs particularly for energy losses less than a few hundred eV, and particularly for heavier and/or thicker specimens which tend to cause strong elastic and thermal diffuse scattering. Fig. 1 shows such an example for chemical maps of a BTO/STO multilayer [1]. For example, in Fig. 1, the original plasmonloss map exhibits strong minima, and the original Ba-N map shows rather poor maxima, both of which are the result of strong elastic scattering.

On the other hand, the largely incoherent conditions lend themselves to a relatively simple procedure to correct, or compensate, for these elastic scattering effects. The "correction map" is derived from the zero- and low-loss intensity, which gives an incoherent bright-field image, the reciprocal of which is taken to obtain the correction map [1]. Despite its simplicity, the procedure has been demonstrated to perform very well under a large range of atomic number and specimen thickness [1,2]. For example, in Fig. 1, the plasmon-loss minima are entirely removed, the chemical contrast of the Ba- $\mathrm{N}$ map is enhanced, and the maps at higher energy losses show only small changes, as expected.

Acknowledgements:

YZ and CD acknowledge financial support from the Australian Research Council (DP110104734). The work at Cornell was supported by the U.S. Department of Energy, Office of Basic Energy Sciences, Division of Materials Sciences and Engineering (DE-SC0002334). 
References:

[1] Y Zhu, A Soukiassian, DG Schlom, DA Muller, C Dwyer, Appl. Phys. Lett. 103 (2013), 141908.

[2] H Tan, Y Zhu, C Dwyer, HL Xin, Phys. Rev. B 90 (2014), 214305.

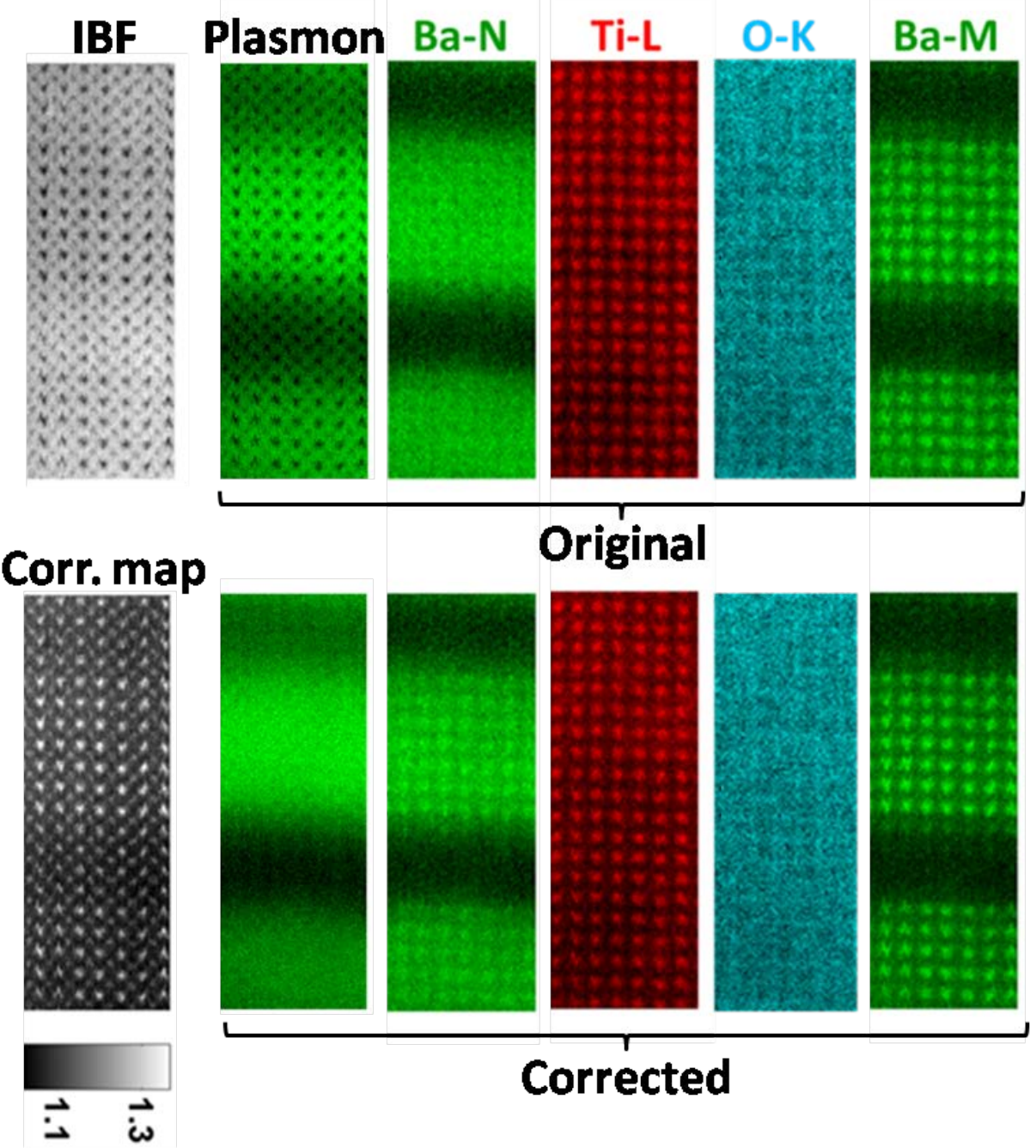

Figure 1. Correction of elastic scattering artifacts in chemical maps of a BTO/STO multilayer. The incoherent bright-field (IBF) and the correction map are shows at left. The color maps at right are the original maps (top row) and corrected maps (bottom row). [Data taken on Nion $100 \mathrm{kV}$ UltraSTEM with a beam convergence semi-angle of $30 \mathrm{mrad}$ and an EELS collection semi-angle of $80 \mathrm{mrad}$. The DualEELS mode of a Gatan Enfinium spectrometer was used: -50-462 eV in 0.5 sec, 320-832 eV in 10 msec]. 